disorders, blue collar with musculoskeletal disorders and inspection with injuries. The findings provide relevant information for disease's prevention and health promotion policies with priority to the most vulnerable occupational groups.

\section{CONDITIONS OF RETURN TO WORK OF NURSES AFTER MATERNITY LEAVE}

Eduardo Sá, Renata Razaboni, Marcela Braga, Flávio Gosling, Marcelo Pustiglione. Faculdade de Medicina Da Universidade de São Paulo, São Paulo, Brazil

\subsection{6/oemed-2014-102362.328}

Objectives Currently, countries like France, Spain, Italy, United States and Brazil there was an increase in the proportion of workers aged between 25-45 years, a period considered fertile. This scenario has determined that many workers had to choose between career and motherhood. The aim is to analyse the amount of Brazilian nursing professionals, in the period from January, 2012 to July 2013, returned to work after maternity leave.

Method Was performed a literature review at Pub Med and BIREME. There was a review of the medical records of nursing professionals met at a public university hospital of São PAulo, Brazil, from January 2012 to July 2013.

Results Returned to work after maternity leave: $80 \%$ (57) of nursing assistants, 70\% (16) of the nursing staff and 94.6\% (53) of nurses. Among the layoffs, $62.5 \%$ (15) occurred within the first 3 months, and only $18.75 \%$ (3) in the first six and nine months after maternity leave.

Conclusions It is possible to conclude that the nursing professional return to work after maternity leave quality requires investment in health policies that promote greater social and labour support.

\section{THE NIEHS GULF STUDY: ESTIMATE OF WORKERS' EXPOSURES THROUGH THE INHALATION ROUTE ON SEVEN RESPONSE VESSELS NEAR THE WELL-SITE DURING THE DEEPWATER HORIZON OIL SPILL}

\footnotetext{
${ }^{1}$ Gurumurthy Ramachandran, ${ }^{1}$ Tran Huynh, ${ }^{2}$ Sudipto Banerjee, ${ }^{2}$ Caroline Groth, ${ }^{3}$ Mark Stenzel, ${ }^{4}$ Aaron Blair, ${ }^{5}$ Lawrence Engel, ${ }^{5}$ Dale Sandler, ${ }^{5}$ Richard Kwok, ${ }^{6}$ Patricia Stewart. 'Department of Environmental Health Sciences, University of Minnesota, Minneapolis, MN, USA; ' Division of Biostatistics, University of Minnesota, Minneapolis, MN, USA; ${ }^{3}$ Exposure Assessment Applications, LLC, Arlington, VA, USA; ${ }^{4}$ National Cancer Institute, Gaithersburg, MD, USA; ${ }^{5}$ Epidemiology Branch, National Institute of Environmental Health Sciences, Research Triangle Park, NC, USA; ${ }^{6}$ Stewart
} Exposure Assessments, LLC, Arlington, VA, USA

\subsection{6/oemed-2014-102362.329}

Objectives After the Deepwater Horizon, response vessels were brought near the wellhead area to stop the leak, collect oil and drill the relief wells. The objective of this paper is to characterise inhalation exposure on these vessels for various exposure groups (EGs) to total hydrocarbons (THCs).

Method Approximately 100 EGs based on job title group, tasks and time period during the response effort were standardised across these vessels. Descriptive statistics were calculated from the measurements for each EG on each vessel. Since many of the data were censored (i.e., below the limit of detection), a Bayesian method was used to obtain estimates for the arithmetic mean (AM), geometric mean, and geometric standard deviation.
Results During the first time period (April 20-May 14), the range of the AMs on the vessels was 1.9-24.2 ppm THC. Exposures were lower during the second period (May 15-July 15) when dispersant was used to reduce air concentrations (0.1-14.6 ppm). After top-capping the well, a substantial reduction was observed on all seven ships (0.1-1.9 ppm). After bottom capping (>August 10), exposures generally increased on the ships (0.1$3.8 \mathrm{ppm})$, most likely due to decontamination activities. The vessel capping the well and the vessel burning waste oil and gas had significant differences from the vessels drilling the relief wells. Conclusions Differences were found by vessel and time period that likely reflected oil weathering and differences in job and vessel activities.

\section{PARENTAL OCCUPATIONAL EXPOSURE TO IONISING RADIATION AND SELECTED BIRTH DEFECTS IN THE US}

${ }^{1} \underline{H}$ Lim, ${ }^{1} \mathrm{AJ}$ Agopian, ${ }^{1} \mathrm{LW}$ Whitehead, ${ }^{2} \mathrm{CW}$ Beasley, ${ }^{3} \mathrm{PH}$ Langlois, ${ }^{1} \mathrm{RJ}$ Emery, ${ }^{1} \mathrm{DK}$ Waller, and The National Birth Defects Preventions Study. 'University of Texas Houston Health Science Center, Houston, USA; ${ }^{2}$ University of Texas School of Medicine, Houston, USA; ${ }^{3}$ Birth Defects Epidemiology and Surveillance Branch, Texas Department of State Health Services, Austin, USA

\subsection{6/oemed-2014-102362.330}

Objectives Results of previous studies of occupational exposure to ionising radiation (IR) and birth defects are inconsistent. Our objective was to assess the association between maternal and paternal occupational exposure to ionising radiation (IR) and birth defects using a larger sample than previous studies.

Method We studied 27809 case mothers and 10200 control mothers who participated in the National Birth Defects Prevention Study (NBDPS), giving birth between 1997 and 2009. Our exposure assessment was based on a textual analysis of the mother's description of her occupation, workplace and job activities (and those of the infant's father) during the three months before and the three months after the estimated date of conception. Logistic regression was used to examine crude and adjusted odds ratios for the association between possible maternal and paternal occupational exposures to IR and 45 birth defects. We assessed the possibility of confounding from pre-pregnancy diabetes and body mass index, smoking, use of supplements containing folic acid, use of alcohol, use of illicit drugs, pregnancy intention, study location and demographic variables.

Results We excluded 17 mothers with a history of cancer, and 12568 mothers who were unemployed, homemakers or students during the periconceptional period. Overall, $3 \%$ of the mothers and $2 \%$ of the fathers were exposed to IR. The remainder of our results are underway and will be presented at the meeting. Conclusions Our results will be interpreted taking account of multiple statistical comparisons and the possibility of recall bias.

\section{MONITORING MERCURY EXPOSURE AMONG ARTISANAL AND SMALL-SCALE MINERS: DEVELOPING AND EVALUATING A SURVEILLANCE PROTOCOL}

Gabriela Gracia, Linda Forst. UIC, Chicago, IL, USA

\subsection{6/oemed-2014-102362.331}

Objectives - Develop a mercury exposure surveillance protocol targeted at artisan and small-scale miners that includes taking occupational histories, ancillary tests, counselling and educating patients regarding exposure 